\title{
Ecuador, microcrédito: ¿Negocio o inclusión financiera?
}

\section{Ecuador, microcredit: Business or financial inclusion?}

\author{
Juanita Salinas Vásquez*
}

\begin{abstract}
Resumen
Una diferencia marcada entre la tasa efectiva que cobran los bancos y organizaciones no gubernamentales que trabajan con microcrédito y la tasa efectiva que cobran las cooperativas de ahorro y crédito, se presentan en este artículo. Cuatro criterios básicos argumentan la situación: la posibilidad de alcanzar economías de escala; la obtención de rendimientos altos; el manejo de costos operativos; y, finalmente, la necesidad de cobertura del riesgo crediticio.
\end{abstract}

\section{Palabras clave}

Financiamiento, economía popular y solidaria, microcrédito, inclusión financiera, emprendimientos populares y solidarios, tasas de interés diferenciadas, metodología de

\section{Resumen}

A matched difference between the effective rate that banks and other not government institutions that work with microcredit charge and the effective rate charged by some saving and credit cooperative is presented here. Four basic criterions argument the situation: the possibility of reaching scale economies, high performance, the handle of operational costs and finally the need of credit risk protection.

\section{Keywords}

Financing, popular and solidarity economy, microcredit, financial inclusion, entrepreneurship and solidarity popular, different interest rates, credit methodology.

Forma sugerida de citar: Salinas, Juanita. 2011. Microcrédito: ¿Negocio o inclusión financiera?. Retos 2. Julio/diciembre. Pp. 39-47.

* Docente de la UPS. Master en Evaluación de Impacto Ambiental. E mail: jsalinas@ups.edu.ec 


\section{Introducción}

El presente documento busca hacer un análisis de las diferentes tasas de interés máximas permitidas a los créditos que se entregan a los agentes de la economía popular (Coragio, 2004: 1-3) con relación a las tasas de interés de los créditos entregados a los agentes de las economías del capital desarrolladas.

Partimos de identificar la magnitud del interés cobrado al compararlo con la inflación y descubrir que la tasa activa es real positiva y alta (superior al 20\%).

Posteriormente valiéndonos de la investigación realizada por la Fundación Mtransparency,[1] la información entregada en la página web de la Superintendencia de Bancos y Seguros (SBS), y la información de campo recolectada en el muestreo y entrevistas realizadas en la investigación "Estructura del Financiamiento de la EPS en el Azuay", se pudo observar una diferenciación de tasas activas en función de dos criterios: del tipo de institución que entrega el crédito, así como del tamaño de crédito que se entrega.

Ante esta situación se establecen al menos tres hipótesis que podrían justificar la tasa diferenciada entre créditos grandes o pequeños, entregados por un tipo de institución u otra.

Las hipótesis son:

a) La diferenciación de tasas genera excedentes o utilidades elevadas, en los créditos dirigidos a las economías populares, por tanto la tasa diferenciada no es más que un mecanismo para extraer importantes ganancias.

Retos 2(1): 2011.

(C) 20II, Universidad Politécnica Salesiana del Ecuador b) El requerimiento de cobrar tasas elevadas para los créditos de actividades productivas de la economía popular está justificado por costos administrativos $\mathrm{u}$ operativos elevados en función del monto de los créditos que se entregan, y de las dificultades de alcanzar economías de escala.

c) La cartera de microcrédito es sensiblemente más riesgosa.

Y por último, una vez salvadas estas hipótesis, se analiza el impacto que tiene el cobro de tasas de interés más elevadas en las economías pequeñas populares.

\section{Objetivos}

La presente busca identificar las causas más relevantes que justifican la tasa diferenciada en los créditos en función de su monto y del tipo de institución que otorga, así como resaltar el impacto que tienen tasas elevadas sobre la población de menores condiciones económicas.

\section{Métodos}

El presente trabajo investigativo sigue el siguiente proceso:

1. Identificación de los principales artículos científicos y documentos relevantes sobre el tema.

2. Identificación y tratamiento de las principales fuentes estadísticas significativas.

3. Levantamiento de las bases de datos para determinar el universo y la muestra de las estructuras organizadas de la economía popular y solidaria.

4. Levantamiento de encuestas a las estructuras organizadas de la EPS 
(154) en el Azuay y entrevistas a los actores más representativos de las instituciones financieras del país y región austral de Ecuador.

5. Tratamiento estadístico de la información recopilada y cruce de información.

6. Sistematización de la información.

Es importante reconocer los límites de la investigación, que está circunscrita a la provincia del Azuay y a las dificultades propias de estudiar un sector con un marco jurídico en construcción y con deficientes estadísticas.

\section{Resultados y discusión}

Las instituciones financieras sean cooperativas o bancos, mantienen tasas reales positivas activas promedio altas en Ecuador, principalmente para los financiamientos a los pequeños emprendimientos, esto significa un ejercicio extractivo fuerte de la economía real. La tasa promedio de crédito microempresa supera el 20\%, mientras la tasa de inflación no supera el 4\%, situación que implica una tasa real activa de más de 16 puntos porcentuales.

Adicionalmente se identifica que existe una sensible diferencia en tasa, sobre todo dependiendo del tipo de institución que otorga los créditos.

Lo expresado es corroborado por el estudio realizado por la fundación Mftrasnparency.org en noviembre de 2010, demostrando que las tasas de interés guardan relación con el tipo de

institución de que se trate: ONG, Banco o Cooperativa, como se expresa en el siguiente gráfico:

Gráfico 1. Instituciones privadas con fines de lucro.

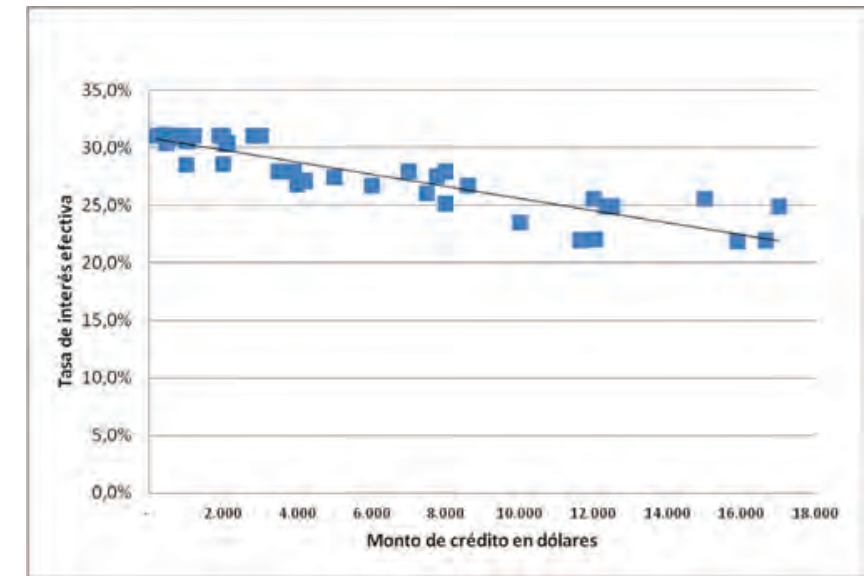

Fuente: 〈http://www.mftransparency.org > Elaboración: Autora. 

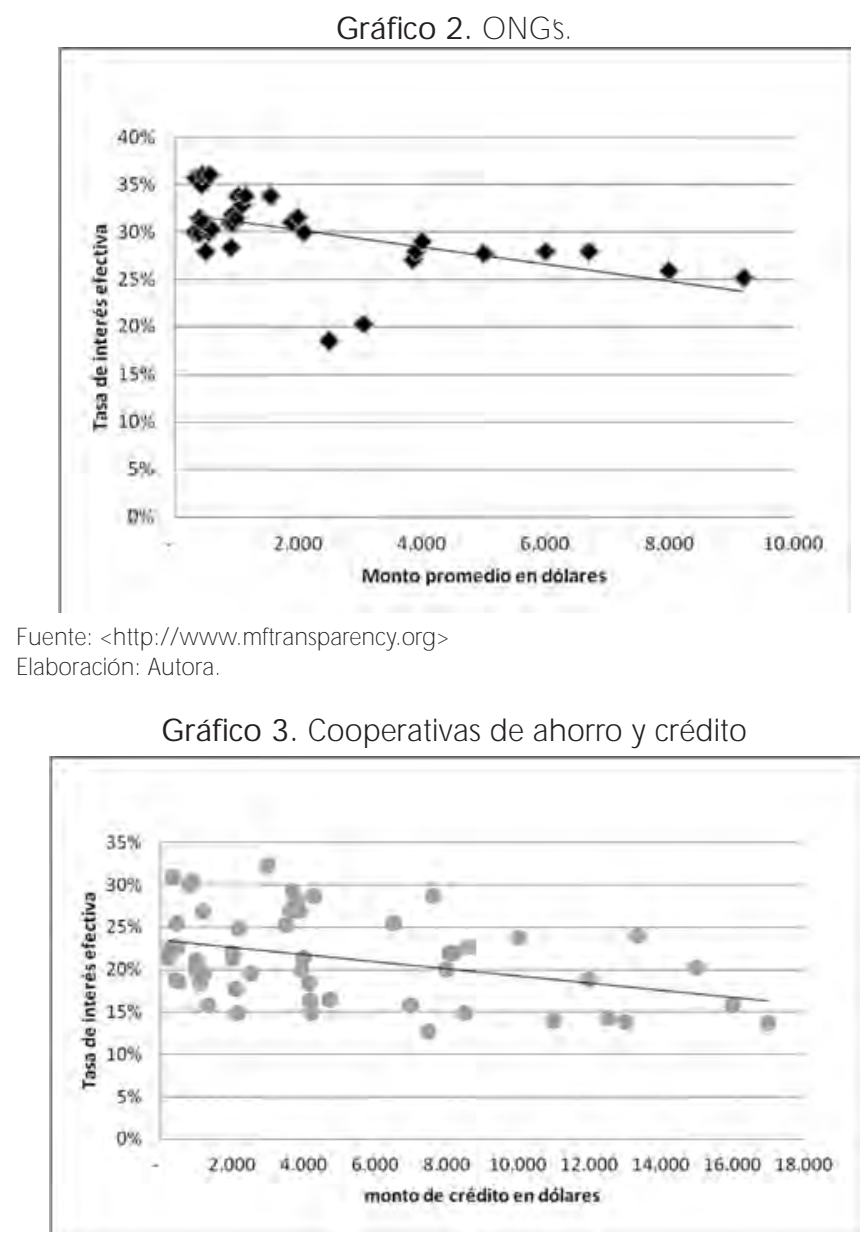

Fuente: «ttp://www.mftransparency.org> Elaboración: Autora.

Las justificaciones respecto a las condiciones diferentes de tasas de interés se suelen esgrimir por al menos cuatro razones:

1. Escala de montos

2. Márgenes elevados de rendimiento del sector

3. Costos Operativos altos

4. Niveles de riesgo elevados

\section{Escala de montos}

La justificación aparentemente más adecuada está centrada en la economía de escala que logran los créditos con su tamaño, esto es diluir los costos fijos al dar montos más altos de crédito.

Sin embargo se puede observar que sin importar el tamaño del crédito las Cooperativas ofertan crédito por 
debajo del promedio y que tanto $\mathrm{ONG}$ que dan crédito como los bancos privados están por sobre el promedio. Esta característica se mantiene en número de instituciones, productos que ofrecen y volumen de cartera colocada.

Lo expresado pone de manifiesto el criterio con el que se estructuran los costos en las instituciones. Para los bancos y ONG's el análisis marginal obliga a ver a cada sujeto beneficiario como pagador de los costos de su crédito. Por el contrario en las cooperativas (principalmente, no en todas) el análisis está basado en el costo medio, situación que permite que los socios que piden créditos más grandes contribuyan a pagar los costos de los socios con créditos más pequeños, logrando con ello un acto cooperativo en el que el grande apoya al pequeño hasta que pueda desarrollarse.
Sin embargo de lo planteado es posiblemente que la diferenciación expresada en párrafos anteriores respecto a la forma en que cobran interés tanto ONG's, bancos y cooperativas, esté marcada también por la metodología de entrega de créditos, por la ubicación o cercanía con el beneficiario, por el costo de fondeo y por el rendimiento exigido por el capital.

\section{Márgenes de rendimiento elevados en el sector}

Otro de los elementos que se esgrime para justificar la diferencia de tasas en discusión son los excedentes o utilidades elevadas. Al respecto se ha procedido a recoger la evolución de los ratios de rentabilidad (ROA y ROE) y su evolución en un período significativo de tiempo con los siguientes resultados:

Tabla 1.

\begin{tabular}{|c|c|c|c|c|}
\hline & $\begin{array}{l}\text { Bancos } \\
\text { grandes }\end{array}$ & $\begin{array}{c}\text { Bancos } \\
\text { Medianos }\end{array}$ & $\begin{array}{c}\text { Bancos } \\
\text { Pequeños }\end{array}$ & $\begin{array}{c}\text { Bancos } \\
\text { Privados } \\
\text { Microempresa }\end{array}$ \\
\hline $\begin{array}{l}\text { Fechas } \\
\text { de corte }\end{array}$ & $\begin{array}{c}\text { Rentabilidad } \\
\text { sobre el } \\
\text { patrimonio }\end{array}$ & $\begin{array}{c}\text { Rentabilidad } \\
\text { sobre el } \\
\text { patrimonio }\end{array}$ & $\begin{array}{c}\text { Rentabilidad } \\
\text { sobre el } \\
\text { patrimonio }\end{array}$ & $\begin{array}{c}\text { Rentabilidad } \\
\text { obre el } \\
\text { patrimonio }\end{array}$ \\
\hline $31 / 12 / 2004$ & $18,2 \%$ & $21,9 \%$ & $28,9 \%$ & $24,4 \%$ \\
\hline $31 / 12 / 2006$ & $26,4 \%$ & $27,2 \%$ & $10,6 \%$ & $6,5 \%$ \\
\hline $31 / 12 / 2007$ & $23,5 \%$ & $19,1 \%$ & $10,0 \%$ & $3,6 \%$ \\
\hline $31 / 12 / 2008$ & $28,9 \%$ & $21,3 \%$ & $14,8 \%$ & $8,4 \%$ \\
\hline $31 / 12 / 2009$ & $14,9 \%$ & $12,7 \%$ & $6,9 \%$ & $6,3 \%$ \\
\hline $31 / 12 / 2010$ & $16,1 \%$ & $16,0 \%$ & $5,0 \%$ & $9,8 \%$ \\
\hline
\end{tabular}

Fuente: Superintendencia de Bancos y Seguros (SBS).

Elaboración: Autora.

Se puede observar que en el período que va desde el 2004 al 2010, los controles de tasas de interés (2007) y los efectos de la crisis financiera internacional han sido absorbidos de mejor manera por los bancos grandes, quienes casi en el períoRetos 2(I): 201 I

(C) 201 I, Universidad Politécnica Salesiana del Ecuador do han podido sostener sus índices de rendimiento, mientras que los pequeños han visto caer fuertemente los mismos.

Para el caso de las cooperativas la situación se presenta de la siguiente manera: 
Tabla 2.

\begin{tabular}{|c|c|c|c|c|c|c|}
\hline & $\begin{array}{l}\text { Coop. } \\
\text { Grandes }\end{array}$ & $\begin{array}{c}\text { Coop. } \\
\text { Medianas }\end{array}$ & $\begin{array}{c}\text { Coop. } \\
\text { Pequeñas }\end{array}$ & $\begin{array}{l}\text { Coop. } \\
\text { Muy pe- } \\
\text { queñas }\end{array}$ & $\begin{array}{l}\text { Coop. } \\
\text { Consumo }\end{array}$ & $\begin{array}{l}\text { Coop. } \\
\text { Micro } \\
\text { credito }\end{array}$ \\
\hline $\begin{array}{l}\text { Fechas } \\
\text { de corte }\end{array}$ & $\begin{array}{c}\text { Rentabilidad } \\
\text { sobre el } \\
\text { patrimonio }\end{array}$ & $\begin{array}{c}\text { Rentabilidad } \\
\text { sobre el } \\
\text { patrimonio }\end{array}$ & $\begin{array}{c}\text { Rentabilidad } \\
\text { sobre el } \\
\text { patrimonio }\end{array}$ & $\begin{array}{l}\text { Rentabilidad } \\
\text { sobre el } \\
\text { patrimonio }\end{array}$ & $\begin{array}{l}\text { Rentabilidad } \\
\text { sobre el } \\
\text { patrimonio } \\
\end{array}$ & $\begin{array}{c}\text { Rentabilidad } \\
\text { sobre el } \\
\text { patrimonio }\end{array}$ \\
\hline $31 / 12 / 2004$ & $11,55 \%$ & $20,07 \%$ & $\mathrm{Nd}$ & $5,52 \%$ & $12,84 \%$ & $23,80 \%$ \\
\hline $31 / 12 / 2006$ & $11,79 \%$ & $9,56 \%$ & $10,64 \%$ & $2,79 \%$ & $6,53 \%$ & $12,58 \%$ \\
\hline $31 / 12 / 2007$ & $9,35 \%$ & $9,50 \%$ & $9,01 \%$ & $5,58 \%$ & $9,10 \%$ & $8,89 \%$ \\
\hline $31 / 12 / 2008$ & $4,60 \%$ & $11,14 \%$ & $10,17 \%$ & $5,61 \%$ & $5,99 \%$ & $10,11 \%$ \\
\hline $31 / 12 / 2009$ & $6,99 \%$ & $10,78 \%$ & $10,16 \%$ & $10,09 \%$ & $7,15 \%$ & $10,87 \%$ \\
\hline $31 / 12 / 2010$ & $10,12 \%$ & $10,67 \%$ & $11,12 \%$ & $3,75 \%$ & $9,44 \%$ & $11,08 \%$ \\
\hline
\end{tabular}

Fuente: Superintendencia de Bancos y Seguros (SBS).

Elaboración: Autora.

Las cooperativas han sufrido en alguna medida también los efectos de las leyes que controlan las tasas de interés y los costos de los servicios. Esta situación es más notoria para las cooperativas medianas y muy pequeñas, $\mathrm{y}$ de especial manera para aquellas que tenían una cartera principalmente enfocada a la microempresa.
Otro aspecto importante que es necesario destacar se puede ver al analizar el margen financiero bruto, análisis que pone de manifiesto el nivel diferenciado de la rentabilidad basado principalmente en el costo bajo de las captaciones que presentan las instituciones grandes.

Así se aprecia en el Gráfico 2:

Gráfico 2. Tasas cobradas y pagadas a microcréditos por los bancos en Ecuador a mayo de 2011

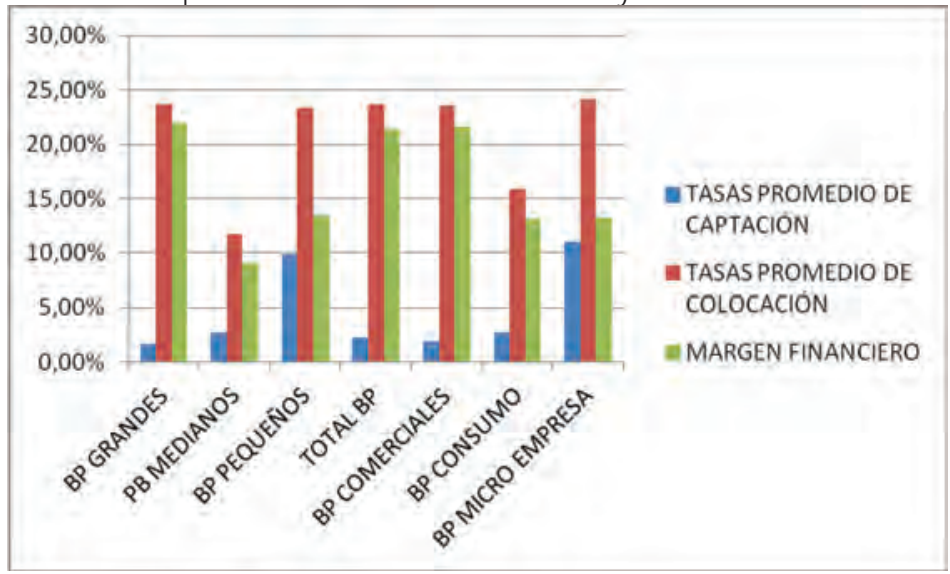

Fuente: Superintendencia de Bancos y Seguros (SBS). Elaboración: Autora. 
Las instituciones grandes tienen por tanto mejores condiciones que las pequeñas en cuanto a rentabilidad bruta causado principalmente por diferenciales sensibles en sus costos financieros.

\section{Costos operativos altos}

En lo referente a la evolución de los costos administrativos, se puede observar un importante esfuerzo (20\%) por reducirlos, especialmente por parte de los bancos medianos y pequeños tal como se aprecia en la siguiente Tabla 3:

Las cooperativas por su parte, también han logrado incrementar su eficiencia operativa, sobre todo las de menor tamaño, situación reflejada en las siguientes cifras:

A pesar del importante esfuerzo de las instituciones financieras nacionales, por incrementar la eficiencia operativa de su gestión, la reducción de costos no ha logrado compensar la contracción en su rentabilidad, generada por la aplicación de las reformas que la "Ley de Justicia Financiera” de junio 2007 estableció para todo el sistema financiero nacional. Dicha ley llevó a una reducción significativa de los límites máximos a las tasas activas y costos de los servicios financieros ofertados.

Tabla 3. Bancos privados de Ecuador. Relación gastos operacionales sobre activos promedios.

\begin{tabular}{|ccccc} 
Fechas de corte & $\begin{array}{c}\text { Bancos } \\
\text { grandes }\end{array}$ & $\begin{array}{c}\text { Bancos } \\
\text { medianos }\end{array}$ & $\begin{array}{c}\text { Bancos } \\
\text { pequeños }\end{array}$ & $\begin{array}{c}\text { Bancos privados } \\
\text { microempresa }\end{array}$ \\
\hline $31 / 12 / 2004$ & $6,6 \%$ & $7,3 \%$ & $12,9 \%$ & $13,1 \%$ \\
\hline $31 / 12 / 2006$ & $5,9 \%$ & $7,6 \%$ & $10,0 \%$ & $12,8 \%$ \\
\hline $31 / 12 / 2007$ & $6,2 \%$ & $6,7 \%$ & $9,2 \%$ & $10,2 \%$ \\
$31 / 12 / 2008$ & $5,5 \%$ & $6,2 \%$ & $6,8 \%$ & $9,7 \%$ \\
$31 / 12 / 2009$ & $5,5 \%$ & $5,3 \%$ & $8,5 \%$ & $9,4 \%$ \\
$31 / 12 / 2010$ & $5,3 \%$ & $4,9 \%$ & $8,4 \%$ & $10,0 \%$ \\
\hline
\end{tabular}

Fuente: Superintendencia de Bancos y Seguros (SBS). Boletín Estadístico.

Elaboración: Autora.

Tabla 4. Cooperativas de ahorro y crédito bajo el control de la SBS. Relación gastos operacionales sobre activos promedios.

\begin{tabular}{ccccccc}
$\begin{array}{c}\text { Fechas de } \\
\text { corte }\end{array}$ & $\begin{array}{c}\text { Coop. } \\
\text { Grandes }\end{array}$ & $\begin{array}{c}\text { Coop. } \\
\text { Medianas }\end{array}$ & $\begin{array}{c}\text { Coop. } \\
\text { Pequeñas }\end{array}$ & $\begin{array}{c}\text { Coop. Muy } \\
\text { pequeñas }\end{array}$ & $\begin{array}{c}\text { Coop. } \\
\text { Consumo }\end{array}$ & $\begin{array}{c}\text { Coop. Mi- } \\
\text { cro credito }\end{array}$ \\
\hline $31 / 12 / 2004$ & $5,80 \%$ & $9,00 \%$ & $0,00 \%$ & $16,10 \%$ & $8,79 \%$ & $7,84 \%$ \\
\hline $31 / 12 / 2006$ & $7,58 \%$ & $6,17 \%$ & $9,73 \%$ & $10,96 \%$ & $4,99 \%$ & $10,29 \%$ \\
\hline $31 / 12 / 2007$ & $6,79 \%$ & $6,02 \%$ & $8,03 \%$ & $13,57 \%$ & $7,14 \%$ & $6,43 \%$ \\
\hline $31 / 12 / 2008$ & $5,09 \%$ & $7,59 \%$ & $7,07 \%$ & $10,29 \%$ & $7,50 \%$ & $6,02 \%$ \\
\hline $31 / 12 / 2009$ & $4,97 \%$ & $5,72 \%$ & $6,25 \%$ & $9,73 \%$ & $5,87 \%$ & $5,65 \%$ \\
\hline $31 / 12 / 2010$ & $5,20 \%$ & $5,69 \%$ & $6,64 \%$ & $9,92 \%$ & $5,75 \%$ & $6,32 \%$
\end{tabular}

Fuente: Superintendencia de Bancos y Seguros (SBS). Boletín Estadístico.

Elaboración: Autora.

Retos 2(1): 2011.

(C) 201 I, Universidad Politécnica Salesiana del Ecuador 


\section{Niveles de riesgo elevados}

Al revisar la variación en el nivel de riesgo de cartera de microcrédito es importante mencionar que no existe una diferencia sustancial en la calificación dada a las instituciones bancarias y a las cooperativas de ahorro y crédito; este indicador si bien explica la tendencia a fijar tasas activas más altas para el sector microempresarial, no constituye una razón para la existencia de tasas diferenciadas entre las instituciones del sistema financiero tradicional y las del sector de la economía popular y solidaria (Gráfico 3).
Aunque la calificación de riesgo de microcrédito es más o menos similar en los diferentes tipos de instituciones financieras, es importante destacar su tendencia a la baja, con excepción de mediados de abril del 2011 cuando presenta una sensible alza (Tabla 5).

\section{Conclusiones}

Las Cooperativas de Ahorro y Crédito han desarrollado un sistema solidario en la gestión de las tasas de interés activas, logrando a través de este procedimiento transferir recursos de los socios más pudientes a los que menos tienen.

Gráfico 3. Evolución del riesgo de la cartera microcrédito

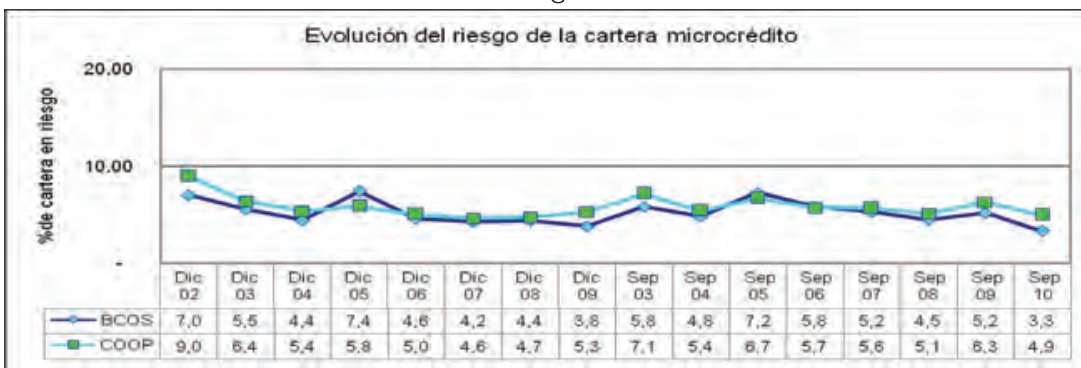

Fuente: Superintendencia de Bancos y Seguros (SBS). Boletín Estadístico. Elaboración: Autora.

Tabla 5. Índices de morosidad de sistema bancario privado

\begin{tabular}{|c|c|c|c|}
\hline TIPOS DE CRÉDITO & $31 / 12 / 2008$ & $31 / 12 / 2009$ & $31 / 12 / 2010$ \\
\hline Comercial & 1,97 & 2,25 & 1,53 \\
\hline Consumo & 4,13 & 4,18 & 3,44 \\
\hline Vivienda & 1,31 & 1,55 & 1,48 \\
\hline Microempresa & 4,84 & 3,82 & 2,63 \\
\hline Total & 2,74 & 2,88 & 2,25 \\
\hline
\end{tabular}

Fuente. Superintendencia de Bancos y Seguros (SBS). Boletines Estadísticos.

Elaboración: Autora. 
Los costos de los créditos están sensiblemente afectados por las tasas de captación o fondeo de recursos de las instituciones financieras. Las entidades más grandes captan recursos a tasas pasivas relativamente más bajas que las entidades financieras pequeñas, ampliando el margen de intermediación y transfiriendo a sus utilidades los ingresos obtenidos.

Las políticas de fijación de las tasas de interés, bajo el criterio de la economía de capital, son un poderoso instrumento de extracción de valor, que acentúa las condiciones de marginalidad y exclusión social.

\section{Recomendaciones}

Impulsar el desarrollo de instituciones financieras populares y solidarias que promuevan la inclusión social y la redistribución de la riqueza

Facilitar la provisión de líneas de recursos adecuados a las instituciones financieras que trabajan con el sector de la economía popular y solidaria, asegurando que dicho beneficio contribuya efectivamente al mejoramiento de las condiciones crediticias de los sectores más necesitados de la economía ecuatoriana.

\section{Notas}

[1] MFTransparency fue creado para proteger el bienestar de los microempresarios de bajos recursos, y para promover la integridad de las microfinanzas como una herramienta para el alivio de la pobreza.

\section{Bibliografía}

Textos

Coraggio, José Luis, "Una Alternativa socioeconómica necesaria: Economía Social”. Debates Fundamentales, Buenos Aires, AltamiraUNGS, 2004.

Salinas Juanita, "Estructura de Financiamiento de la Economía Social y solidaria en la provincia del Azuay" Proyecto de investigación Universidad Politécnica Salesiana, 2011.

Sitios web

Superintendencia de Bancos y Seguros del Ecuador, mayo del 2011, "Catastro de instituciones", en línea $<\underline{\text { www.superban.gov.ec }>}$

Superintendencia de Bancos y Seguros, mayo del 2011. Concentración de Activos, en línea $<$ www.superban.gov.ec $>$

Banco Central del Ecuador, Abril 2011 "Evolución del Crédito y Tasas Efectivas Referenciales. Dirección de Estadísticas Económicas", en línea $<\underline{w w w} \cdot$ bce.fin.ec $>$

MTransparency, noviembre 2010, "Tasas de interés por institución, destino y monto" $<$ http://www.mftransparency.org $>$

Envío 14/julio/2011 - Aceptación 10/noviembre/2011

Retos 2(I): 201I.

(C) 20II, Universidad Politécnica Salesiana del Ecuador 